\title{
Comparison of videonasoendoscopy and auditory-perceptual evaluation of speech in individuals with cleft lip/palate
}

\author{
Lauren Medeiros Paniagua ${ }^{1}$, Alana Verza Signorini², Sady Selaimen da Costa ${ }^{3}$ Marcus Vinicius Martins Collares ${ }^{4}$, \\ Sílvia Dornelles 5 .
}

1) Speech Language Pathologist. Doctor of Science in Children's and Teenager's Health- Universidade Federal do Rio Grande do Sul-UFRGS. Professor of Speech-Language Pathology at Fatima College (RS).

2) Undergraduated Speech pathology - Universidade Federal do Rio Grande do Sul - UFRGS (fellow undergraduated of CNPq).

3) MD; MSc; PhD. Associate Professor - Department Of Otolaryngology \& Head And Neck Surgery School Of Medicine - Universidade Federal do Rio Grande do Sul.

4) MD; PhD. Associate Professor Department of Surgery; Head, Plastic Surgery Section - School of Medicine/Universidade Federal do Rio Grande do Sul.

5) Speech Pathology, MSc, PhD. Professor Department of Speech Pathology - Universidade Federal do Rio Grande do Sul.

Institution: Universidade Federal do Rio Grande do Sul (UFRGS)

Porto Alegre / RS - Brazil.

Mailing address: Lauren Medeiros Paniagua - Universidade Federal do Rio Grande do Sul (UFRGS) - Avenida João Wallig $1705 / 627$ - Porto Alegre / RS - Brazil - Zip Code 91340-001 -E-mail: lmedeirospaniagua@yahoo.com.br

Article received on December $17^{\text {th }}, 2012$. Article accepted on April $7^{\text {th }}, 2013$.

\section{SUMMARY}

Introduction: The velopharyngeal sphincter (VPS) is a muscle belt located between the oropharynx and the nasopharynx. Investigations of velopharyngeal function should include an auditory-perceptual evaluation and at least 1 instrument-based evaluation such as videonasoendoscopy.

Aim: To compare the findings of auditory-perceptual evaluation (hypernasality) and videonasoendoscopy (gap size) in individuals with cleft lip/palate.

Method: This was a retrospective, cross-sectional study assessing 49 subjects, of both sexes, with cleft lip/palate followed up at the Otorhinolaryngology Service and the Speech Therapy outpatient clinic of Hospital de Clínicas de Porto Alegre (HCPA). The results from the auditory-perceptual evaluation and the videonasoendoscopy test were compared with respect to the VPS gap size.

Results: Subjects with moderate/severe hypernasality had more severe velopharyngeal closure impairment than those with a less severe condition. The interaction between hypernasality severity and the presence of other speech disorders ( $\mathrm{p}=0.035$ ), whether compensatory and/or obligatory, increased the likelihood of having a moderate-to-large gap in the velopharyngeal closure.

Conclusions: We observed an association between the findings of these 2 evaluation methods.

Keywords: Cleft Palate; Velopharyngeal Sphincter; Communication Disorders; Evaluation.

\section{INTRODUCTION}

The velopharyngeal sphincter (VPS) is a muscle belt located between the oropharynx and the nasopharynx and includes the muscles of the soft palate (anterior wall of the VPS) and the lateral and posterior pharyngeal walls. The VPS comprises the levator veli palatini, tensor veli palatini, muscle of uvula, superior pharyngeal constrictor, palatopharingeus, palatoglossus, and salpingopharyngeus (1). Among these muscles, those of the soft palate, especially the tensor veli palatine, have an important role in the physiological function of the region. While the VPS is not a sphincter by strict definition (a circular muscle band in a hollow viscera), its physiological function is that of a sphincter, as it works like a valve, contracting and occluding itself (2).

The VPS plays an extremely important role in allowing alternation between the respiratory and digestive paths of the pharynx during swallowing (3). Velopharyngeal closure, performed by the VPS, is essential in motor actions of the region such as speaking, whistling, blowing, sucking, and swallowing (4). Several studies have described intersubject variability in velopharyngeal closure $(1,5)$ and have identified 4 types of closure based on structure movement $(6,7,1,5)$. In the coronal type of closure, there is more participation of the soft palate. Sagittal closure predominantly involves the lateral pharyngeal walls. In the circular type of closure, the soft palate and lateral pharyngeal walls are involved. Finally, the circular type closure with a Passavant ridge, is similar to the circular closure except that a Passavant ridge is present on the posterior pharyngeal wall.

The configuration of the velopharyngeal region depends on the specific motor actions performed. The raising of the soft palate and, concomitantly, the medial approximation of the lateral pharyngeal walls promotes the partial or complete separation of the nasal and oral 
portions of the pharynx. The velopharyngeal structures are thus important for the production of speech, as they distribute and direct airflow to the oral cavity to produce oral sounds and to the nasal cavity for the emission of nasal sounds $(1,8,9)$.

Investigators have several types of tools to choose from when assessing VPS function (10, 11, 12). A detailed description of the velopharyngeal mechanism was obtained only after the availability of both direct and indirect assessment methods. Direct methods enable the investigator to visualize the structures involved in velopharyngeal closure and to observe how these structures move during different types of motor actions. Indirect methods provide information on the functional repercussions of velopharyngeal action $(13,12,14)$.

Videonasoendoscopy is one of the most widely used methods for studying velopharyngeal function, allowing visualization of the nasal, pharyngeal, and laryngeal cavities. Using dynamic images directly from the anatomical structures (15), this method has multiple applications, including diagnosis, prognosis, and postoperative follow up (11). This test can be used to observe the pattern of closure even during speech, which involves wide variations in velum and pharyngeal wall motion. Videonasoendoscopy can also be used to identify the presence of the gap corresponding to the residual orifice during maximum contraction of the VPS $(16,17)$.

As a counterpart to the use of more sophisticated tools for velopharyngeal assessment, clinical judgment by means of hearing is still considered an extremely important diagnostic tool for inferring velopharyngeal function (12). Using this method, the investigator can assess a subject's oral communication performance and determine the extent of speech impairments related to resonance $(18,12,19$, 20, 21).

Impaired velopharyngeal closure prevents separation of the oral and nasal cavities during the production of oral phonemes, exposing the nasal cavity to the entry of unexpected air flow. This impairment in velopharyngeal closure, known as velopharyngeal dysfunction (VPD), can be congenital, as with cleft lip.

There is a widespread consensus among researchers and healthcare professionals that the surgical and clinical findings of subjects with cleft lip/palate should be based on an auditory-perceptual evaluation and at least one instrument-based evaluation of velopharyngeal function $(13,22,23,24)$. The use of both assessment modalities is highly valuable and widely used in clinical practice, as the findings of one method complement the other. Despite this common practice, there are few studies comparing patient evaluations using videonasoendoscopy (clinical estimation of gap size) and auditory-perceptual assessments (hypernasality). This study aimed to determine whether the findings of videonasoendoscopy in patients with cleft lip/palate are associated with demographic features, clinical factors, or the findings of auditory-perceptual assessments.

\section{Methods}

This retrospective, cross-sectional study assessed 49 individuals, of both genders, with cleft lip/palate who were followed up at the Otorhinolaryngology Service and at the Speech Therapy outpatient clinic of Hospital de Clínicas de Porto Alegre (HCPA). The study was approved by the HCPA Research Ethics Committee under protocol number 10-0490.

The population consisted of individuals follow up at the HCPA in the Plastic Surgery Service, Craniofacial Surgery outpatient clinic; Otorhinolaryngology and at the "SpeechTherapy and Craniofacial Malformations" outpatient clinic. This group of patients was primarily treated at HCPA and at other institutions.

The present study compared the results of 2 methods for evaluating velopharyngeal function. The auditoryperceptual evaluation by speech therapy screening is reported as speech findings. This assessment was carried out by a speech therapist with more than 10 years of experience in the area. The results of videonasoendoscopy were analyzed by 2 experienced evaluators. The speech therapy screening and videonasoendoscopy data were collected in October 2012. The inclusion criterion was assessment by speech therapy screening and videonasoendoscopy between September 2011 and August 2012. We excluded from the study patients with blurred videonasoendoscopy images and those with excess secretion from the nasal cavity or missing data.

The speech therapy screening assessed the following auditory-perceptual parameters: resonance, presence, and severity of hyponasality and hypernasality (mild, moderate, severe) (25); speech disorders, identified as compensatory (such as glottal stops) or obligatory (weak intraoral pressure, facial mimicry, audible nasal air emission, nasal snoring). For analysis purposes, hypernasality and its severity were included as obligatory disorders (26). During screening, the speech therapist used a speech sample comprising 2 sentences with phonetic predominance of oral plosive phonemes/p/ ("papai pediu pipoca") and fricative phonemes/s/ ("o saci sabe assobiar" The patients also performed a segment of continuous speech, counting from 1 to 10 . 
Using videonasoendoscopy, we analyzed the portion of the test related to the sustained emission of the $/ \mathrm{s}$ / phoneme, chosen because it leads to complete closure of the VPS, thereby minimizing misinterpretations due to interference by the production of another phoneme. The evaluators made a clinical estimation of the VPS gap size using an adapted version of a protocol described in previous studies $(10,27)$. The VPS gap size was classified according to the following scale: 1 , no gap (complete velopharyngeal closure); 0.8-0.9, small gap (efficient closure); 0.4-0.7, moderate gap (intermediate closure); 0.1-0.3 large gap (inefficient closure); 0 , very large gap (lack of closure). This protocol assessed the following: presence or absence of adenoids contributing to velopharyngeal closure; presence or absence of mobility of the palate and lateral and posterior pharyngeal walls; VFS closure type (coronal, sagittal, circular, or circular with Passavant ridge).

Quantitative variables were described as mean and standard deviation or median and interquartile range. Categorical variables were described as absolute or relative frequencies. The Pearson's chi-square test was used to evaluate the association between videonasoendoscopy and perceptual-hearing evaluation. The McNemar's test was applied to assess the differences between evaluators, and the kappa coefficient was applied to assess interevaluator agreement. Student's $t$-test (quantitative variables with symmetric distribution), Mann-Whitney test (quantitative variables with asymmetric distribution), and Pearson's chi-square and Fisher's exact tests (categorical variables) were applied to investigate the association between clinical factors and examination methods. Poisson's regression model was used to control confounding factors. The criterion for including a variable in the model was a $\mathrm{p}$ value $<0.10$ in the bivariate analysis. We considered significance to be 5\% (pd" 0.05). Analyses were performed using SPSS (Statistical Package for the Social Sciences) version 17.0

\section{RESULTS}

The median age at the time of evaluation was 11 years, with the majority of the cohort between 9 and 16 years. The cohort was predominantly male (61.2\%). Speech therapy treatment was ongoing in $14.3 \%$ of the subjects.

The most common cleft type was the left unilateral complete cleft $(30.6 \%, \mathrm{n}=15)$, followed by bilateral complete cleft lip/palate $(28.6 \%, \mathrm{n}=14)$, right unilateral $(20.4 \%, n=10)$, cleft palate $(14.3 \%, n=7)$, and submucosal cleft $(6.1 \%, n=3)$.

All patients had undergone primary palatoplasty at a mean age of 19.5 months. Secondary palatoplasty was performed in only $4.1 \%$ of the subjects, at a median age of 18.5 years.

Table 1 shows the results from the speech therapy screening, including the assessment of resonance as well as compensatory and obligatory articulation disorders. We found that $77.6 \%$ of the subjects presented with hypernasality, which was moderately severe in $36.7 \%$ and mildly severe in $28.6 \%$ of these subjects. As illustrated in Table 1, articulation disorders were found in $81.6 \%$ of subjects, $75.5 \%$ of whom had obligatory disorders and $57.1 \%$ of whom had compensatory disorder (glottal stops). The most frequently observed obligatory disorder was weak intraoral pressure, which was present in $75.7 \%$ of the subjects. The second more frequent disorder was audible nasal air emission (62.2\%).

Inter-evaluator comparison of the findings of the videonasoendoscopy, as well as comparison of these test results with those of the auditory-perceptual evaluation was assessed using the emission of the $/ \mathrm{s} /$ phoneme.

The inter-rater agreement was established using the Kappa agreement coefficient, matching the findings of one rater with those of the other. In this study, the Fleiss' kappa agreement coefficients (28) were interpreted as previously reported (29): $<0.00$, no agreement; $0.00-0.20$, poor agreement; $0.21-0.40$, fair agreement; 0.41-0.60, moderate agreement; 0.61-0.80, substantial agreement; and $0.81-1.00$, perfect (or almost perfect) agreement.

An almost perfect agreement (kappa of 0.83-1.00) was observed regarding the contribution of adenoids in

Table I. Auditory-perceptual evaluation results*.

\begin{tabular}{|c|c|}
\hline \multicolumn{2}{|l|}{ Speech disorder, n(\%) } \\
\hline Present & $44(89.8)$ \\
\hline Absent & $5(10.2)$ \\
\hline \multicolumn{2}{|l|}{ Type of disorder*, n(\%) } \\
\hline Obligatory & $43(87.8)$ \\
\hline Compensatory & $28(57.1)$ \\
\hline \multicolumn{2}{|c|}{ Types of obligatory disorders*, n(\%) } \\
\hline Audible nasal air emission & $23(62.2)$ \\
\hline Weakintraoral pressure & $28(75.7)$ \\
\hline Facialmimicry & I | (29.7) \\
\hline Nasal snoring & $2(5.4)$ \\
\hline Hypernasality & $38(77.6)$ \\
\hline \multicolumn{2}{|l|}{ Resonance, n(\%) } \\
\hline Balanced & II (22.4) \\
\hline Mildhypernasality & $14(28.6)$ \\
\hline Moderatehypernasality & $18(36.7)$ \\
\hline Severehypernasality & $6(12.2)$ \\
\hline
\end{tabular}


velopharyngeal closure, mobility of pharyngeal walls, predominant type of movement, closure type, and VPS gap size during emission of the /s/ phoneme. The predominant closure type was coronal. One evaluator's results indicated that $20.4 \%$ of the subjects had no VPS gap, $40.8 \%$ had a small gap, $16.3 \%$ had a moderate gap, and $22.4 \%$ had a large gap.

Demographic data indicated that the subjects with more severe velopharyngeal closure impairment underwent later primary palatoplasty; the presence of a moderate to large gap was significantly associated with an age at primary palatoplasty of over 23 months. All individuals requiring secondary palatoplasty $(n=2)$ showed impairment in velopharyngeal closure, although this result was not statistically significant. There were no other statistically significant associations between variables.

We compared the VPS gap size as estimated by clinical and auditory-perceptual assessments (Table 2). We also investigated the relationship between the gap size and both the severity of hypernasality and the presence of speech disorders (compensatory and obligatory disorders).

Our results indicate that more severe impairment of velopharyngeal closure was observed more often in subjects with moderate/severe hypernasality, with statistical significance for severe hypernasality $(p=0.021)$. In addition, we found severe hypernasality was significantly associated with the presence of other speech disorders $(p=0.035)$, whether compensatory and/or obligatory, increasing the likelihood of having a moderate to large gap (intermediate to inefficient velopharyngeal closure).

Ten subjects (20.4\%) showed discrepant results with regard to velopharyngeal function, having moderate/ severe hypernasality + other speech disorders together with complete and efficient gap closure. Conversely, 6 subjects (12.2\%) had intermediate/inefficient closure with no moderate/severe hypernasality.

These discrepant findings may be due to the differences in the analytical parameters (speech and continuous sound emission), although both parameters involve velopharyngeal function. The auditory-perceptual evaluation assessed a larger speech sample (counting of numbers and 2 sentences focusing on $/ \mathrm{s} /$ and $/ \mathrm{p} /$ phonemes). The instrumental evaluation observed the sustained emission of the $/ \mathrm{s} /$ phoneme, which provides maximum VPS contraction. In such cases that present physical evidence of closure but indicate abnormal resonance, it is very important for the interdisciplinary team to re-evaluate the findings to perform a differential diagnosis of velopharyngeal dysfunction. Therefore, it can be inferred that the maintenance of motor action in speech is not consistent.

Table 2. Relationship between auditory-perceptual assessment and gap size.

\begin{tabular}{|c|c|c|c|}
\hline Variables & Complete/efficientclosure & Intermediate/inefficientclosure & $\mathrm{p}$ \\
\hline Speech disorder, n(\%) & & & 0.636 \\
\hline Present & $26(86.7)$ & $18(94.7)$ & \\
\hline Absent & $4(13.3)$ & I (5.3) & \\
\hline \multicolumn{4}{|l|}{$\begin{array}{l}\text { Moderate/severe hypernasality + } \\
\text { otherspeech disorders, } \mathrm{n}(\%)\end{array}$} \\
\hline & & & 0.035 \\
\hline Yes & $10(33.3)$ & $13(68.4)$ & \\
\hline No & $20(66.7)$ & $6(31.6)$ & \\
\hline Type of disorder, n(\%) & & & 0.157 \\
\hline Obligatory & $12(46.2)$ & $4(22.2)$ & \\
\hline Compensatory & I (3.8) & $0(0.0)$ & \\
\hline Obligatory + compensatory & $13(50.0)$ & $14(77.8)$ & \\
\hline \multicolumn{4}{|l|}{ Types of obligatory disorders*, n(\%) } \\
\hline Audible nasal airemission & $13(43.3)$ & $10(52.6)$ & 0.733 \\
\hline Weakintraoral pressure & $17(56.7)$ & || (57.9) & 1.000 \\
\hline Facialmimicry & $4(13.3)$ & $7(36.8)$ & 0.081 \\
\hline Nasal snoring & $2(6.7)$ & $0(0.0)$ & 0.515 \\
\hline Hypernasality & $21(70.0)$ & $17(89.5)$ & 0.165 \\
\hline Resonance, n(\%) & & & 0.021 \\
\hline Balanced & $9(30)$ & $2(10.5)$ & \\
\hline Mild hypernasality & II (36.7) & $3(15.8)$ & \\
\hline Moderatehypernasality & $9(30)$ & $9(47.4)$ & \\
\hline Severehypernasality & I (3.3) & $5(26.3)$ *** & \\
\hline
\end{tabular}

*multiple choice question; ***tatistically significant association by the test of residuals adjusted to a $5 \%$ of significance level. 


\section{DISCUSSION}

The physiology of the velopharyngeal mechanism has been investigated in several disciplines (30, 31). Studies comparing different methods of assessing velopharyngeal function report conflicting results. Few of these investigations have considered the following factors in the same study: (1) protocols for clinical and instrumentbased assessments recommended in the literature, including a detailed description of the criteria used for analysis of the variables under study; (2) inter-evaluator agreement for assessments using videonasoendoscopy; (3) the possible variables interfering with velopharyngeal closure (participation of adenoids and speech therapy treatment for velopharyngeal occlusion).

Our study assessed hypernasality, compensatory articulation disorders, and other obligatory disorders (26). These speech anomalies are of interest to researchers who investigate speech production in individuals with cleft lip/ palate $(32,3334,35,25)$.

A recently study (26) has emphasized that obligatory disorders should receive attention from a structural point of view and not only from the perspective of velopharyngeal function; the identification of velopharyngeal dysfunction can help to establish whether speech therapy intervention is indicated for individuals with cleft lip/palate. The auditoryperceptual evaluation used in the present study took these structural features of obligatory disorders into account. Hypernasality was reported in $77.6 \%$ of our subjects, the severity of which was moderate in $36.7 \%$ and mild in $28.6 \%$. Speech disorders were identified in $89.8 \%$ of the subjects, of whom $87.8 \%$ had obligatory disorders and $57.1 \%$ compensatory disorder (glottis stop). One of the most frequent obligatory disorders was weak intraoral pressure, present in $75.7 \%$ of the subjects. Our findings corroborate those in the literature reporting a higher occurrence of weak intraoral pressure, hypernasality, and glottis stop $(37,4,37)$. The results of this study thus suggest that the weak intra-oral pressure caused by VPD produces audible nasal air emission and hypernasality in speech. In turn, glottis stops may occur as a consequence of poor articulation habits learned in childhood that do not necessarily reflect physical or neuromuscular changes (38).

We believe that the high occurrence of hypernasality and other articulation disorders in our cohort is related to their referrals from speech therapy, indicating that they had an issue involving oral communication. Although these patients were followed up for a longer time by the HCPA interdisciplinary team that provides a variety of services, a speech therapy outpatient clinic linked to Universidade Federal do Rio Grande do Sul was added in 2011 to treat patients with cleft lip/palate. Before the creation of this outpatient clinic, the patients were treated just by a speech therapist from HCPA, with a period of care limited to one shift for this population. As it constitutes a curricular internship, in which undergraduate and graduate students practice the activity, the response to this demand is still limited.

Reports in the literature point out that experience is crucial in both the conduction and analysis of the videonasoendoscopy test. Accordingly, we assessed the inter-evaluator agreement with regard to gap size in the emission of the $/ \mathrm{s} /$ phoneme, observing that it was almost perfect (kappa, 0.83-0.95). We thus conclude that the evaluation team is highly qualified. This result also illustrates that the criteria adopted for interpreting the findings were very homogeneous between the raters. These findings corroborate those described in other scientific studies using this protocol (10), indicating that the data are in accordance with the proposed objective $(39,40,41)$.

We observed that $20.4 \%$ of the subjects did not have a VPS gap; $40.8 \%$ had a small gap, $16.3 \%$ had a moderate gap, and $22.4 \%$ had a large gap. Thus, $61.2 \%$ of the total cohort had complete or efficient velopharyngeal closure. The predominant velopharyngeal closure type was coronal $(69.4 \%, n=34)$ which has greater mobility of the anterior wall of the VPS corresponding to the soft palate. The prevalence of the sagittal type was $24.5 \%$ (12), and the circular type was $6.1 \%$ (3). Studies have demonstrated that the coronal pattern is also the most frequent among individuals without cleft lip/palate (42, 22, 30, 7), but individuals with cleft palate tend to have a broader distribution between the other patterns of closure $(43,7,44)$.

Among the associations related to demographic and clinical data, only age at the time of primary palatoplasty was associated with the clinical estimation of VPS gap size. Our findings indicate that the later primary palatoplasty is performed the greater the compromise in velopharyngeal closure (moderate to large gap size), with a significant associated with ages above 23 months $(p=0.016)$. However, when analyzing the variables independently associated with intermediate/inefficient velopharyngeal closure (by controlling confounding factors), age at primary palatoplasty was not significantly associated but was near the threshold ( $\mathrm{PR}=1.01 ; 95 \% \mathrm{CI}, 1.00-1.02 ; \mathrm{p}=0.088$ ). The trend in the data indicates that every additional month until the performance of the first palatoplasty leads to a $2 \%$ increase in the prevalence of inefficient closure.

The author of a previous study (46) proposes that the appropriate time for surgical repair of the palate is 12 18 months of age, taking into account maxillary growth and velopharyngeal function. One report on the association between various clinical factors and velopharyngeal closure 
in cleft lip/palate found that velopharyngeal closure was higher among individuals with deciduous dentition than among those with mixed or permanent dentition (47).

The present study aimed to compare the findings of auditory-perceptual evaluation and those of videonasoendoscopy. Our data indicate that subjects with more compromised velopharyngeal closure have a higher incidence of moderate and severe hypernasality, showing that both tests indicated the compromise in velopharyngeal function. A statistically significant association was found between severe hypernasality ( $p=0.021$ ) and intermediate/ inefficient closure with a gap size ranging from moderate to large.

We found one study that aimed to evaluate auditoryperceptual assessment, but the authors adopted a different classification system for the findings of videonasoendoscopy and did not relate them to the efficiency of velopharyngeal closure. A retrospective study investigated the relationship between the auditory-perceptual characteristics of speech and velopharyngeal gap size (20), and the authors concluded that information regarding velopharyngeal gap size can be predicted from speech assessment alone. Confidence in the prediction was stronger when the patient had moderate to severe hypernasality, which was more commonly associated with a large gap. This last finding was similar to that reported in our study.

We found a statistically significant interaction $(\mathrm{p}=$ 0.035 ) of the severity of hypernasality and the presence of other articulation disorders with the clinical estimate of VPS gap size. The interaction of both changes identified in the auditory-perceptual evaluation increased the likelihood of the presence of a moderate to large gap. Multivariate analysis confirmed this association (PR $=1.26$; 95\% CI: $1.03-1.54 ; \mathrm{p}=0.026$ ). This result is observed in the clinical practice, as the presence of hypernasality is usually associated with at least one articulation change, whether obligatory or compensatory. The articulation changes associated with hypernasality reflect the difficulty in acquiring and maintaining velopharyngeal closure (47).

To gain a better understanding of these cases, we investigated velopharyngeal physiology during the motor action related to speech. Although no statistically significant difference was found, 2 interesting situations were observed. First, 10 subjects (20.4\%) had complete/efficient velopharyngeal closure but also had moderate/severe hypernasality. Second, 6 subjects (12.2\%) had intermediate/ inefficient closure with no moderate/severe hypernasality.

Although both videonasoendoscopy and auditoryperceptual evaluation assess velopharyngeal function, this study used different samples and speech extensions. The videonasoendoscopy analyzed only the sustained emission of the $/ \mathrm{s} /$ phoneme, using a comfortable intensity for the patient that was audible for the examiner. This phoneme was chosen because its emission requires the action of all velopharyngeal walls and their maximum contraction to achieve complete velopharyngeal closure. This information allows the examiner to identify the presence or absence of a VPS gap.

Velopharyngeal closure differs during different motor actions and during speech (4). The observation that some patients have unsatisfactory findings in the auditoryperceptual evaluation and good findings in videonasoendoscopy suggests that resonance in continuous speech is unaffected. However, articulation disorders can hamper accurate assessment of hypernasality severity. Hypernasality is often associated with the length of time required for VPS opening and not directly with the degree of opening or even with the air flow that escapes from the nasal cavity (48). For example, patients with adequate velopharyngeal closure as assessed by instrument-based assessment may have hypernasality because of the abnormalities in the temporal spectrum of velopharyngeal closure.

The great physiological variability of the VPS may be related to factors such as speed of speech, characteristics of isolate phoneme emission, and association of different phonemes (consonant-vowel) (1). This variability may account for the patients who have intermediate/inefficient closure but no moderate/severe hypernasality. Further factors may explain this versatility in velopharyngeal closure as follows: the action of several muscles associated with the levator veli palatini (palatoglossus, palatopharingeus, and superior pharyngeal constrictor); mechanical factors, especially the position of the tongue in the oral cavity; and the specific phonological rules of each linguistic system $(49,5)$.

In summary, we recommend that patients with discrepant results between evaluations and those with unsatisfactory results should be referred to interdisciplinary clinical evaluations to determine the best course of action. (26). Xet al. Emphasizes that obligatory and compensatory articulation disorders affect speech, and that obligatory disorders should receive attention from both the structural and functional points of view (26). Therefore, by identifying velopharyngeal dysfunction, appropriate therapeutic strategies can be devised.

\section{Conclusions}

It was found that our patient cohort had a high prevalence of hypernasality and compensatory and 
articulation disorders. In addition, we observed a high frequency of individuals with a small gap, and a predominance of the coronal type closure. The individuals with more severe velopharyngeal closure impairment underwent late palatoplasty, although this comparison did not remain significant after adjustment by the multivariate model.

Comparison of the 2 evaluation methods showed that subjects with moderate/severe hypernasality had more severely impaired velopharyngeal closure. Hypernasality is more closely associated with clinical estimation of gap size than are other obligatory and compensatory disorders. The interaction between hypernasality severity and the presence of other disorders increases the likelihood of having a gap ranging from moderate to large. Although we found cases with discrepancies between the findings of the auditoryperceptual and instrument-based evaluations, we observed an association between the findings of these 2 evaluation methods.

\section{REFERENCES}

1. Altmann EBC. Anatomia e fisiologia do esfíncter velofaríngeo. In: Altmann EBC, editor. Fissuras labiopalatinas. 4 ed. Carapicuíba: Pró-fono; 1997. p. 133-56.

2. Lofiego JL. Fissura Labiopalatina: avaliação, diagnóstico e tratamento fonoaudiológico. Rio de Janeiro: Revinter; 1992.

3. Silva DP, et al. Aspectos patofisiológicos do esfíncter velofaríngeo. Arq. Int. Otorrrinolaringol. 2008;12(3):426-35.

4. Altmann EBC, Ramos ALNF, Khoury RBF. Avaliação Fonoaudiológica. In: Altmann EBC. Fissuras Labiopalatinas. Carapicuíba: Pró- Fono; 2005. p. 325-66.

5. Finkelstein Y, et al. Axial Configuration of velopharyngeal valve and its valving mechanism. Cleft Palate Craniofac J. 1995;32:299-305.

6. Skolnick ML, Mccall GN, Barnes M. The sphincter mechanism of velopharyngeal closure. Cleft Palate J. 1973;10:286-305.

7. Croft CB, Shprintzen RJ, Rakoff SJ. Patterns of velopharyngeal valving in normal and cleft palate subjects: a multi-view videofluoroscopic and nasendoscopic study. Laryngoscope. 1980;91:265-71.

8. Shprintzen RJ, BardachJ. Cleft palate Speech management: A Multidisciplinary Approach. New York: Mosby; 1995.
9. Trindade IEK, Trindade Junior AS. Avaliação funcional da inadequação velofaríngea. In: Carreirão S, Lessa S, Zanini AS, editors. Tratamento das fissuras labiopalatinas. $2 \mathrm{ed}$. Rio de Janeiro: Revinter; 1996. p. 223-35.

10. Golding-Kushner KJ, et al. Standartization for the reporting of nasopharyngoscopy and multiview videofluoroscopy: a report from an international working group. Cleft Palate J. 1990;27:337-48.

11. Rocha DL. Insuficiência Velofaríngea. In: Mélega JM. Cirurgia Plástica- Fundamentos e Arte-Cirurgia reparadora de Cabeça e Pescoço. Rio de Janeiro: Medsi, 2002. p. 17896.

12. Genaro KF,Yamashita RP, Trindade IKE. Avaliação clínica e instrumental na fissura labiopalatina. In: Ferreira LP, BefiLopes DM, Limongi SCO. Tratado de Fonoaudiologia. São Paulo: Rocca; 2004. p. 456-77.

13. Dalston RM, Warren DW, Dalston ET. Use of nasometry as a diagnostic tool for identifying patients with velopharyngeal impairment. Cleft Palate-Craniofacial J. 1991;28:184-9.

14. Nakamura N, Ogata Y, Sasaguri M, Suzuki A, Kikuta R, Ohishi. Aerodynamic and cephalometric analyses of velopharyngeal structure and function following repushback surgery for secundary correction in cleft palate. Cleft Palate Craniofac J. 2003:40:46-53.

15. Pontes PAL, Behlau MS. Nasolaringoscopia. In: Altmann EBC. Fissuras labiopalatinas. 4th ed. Carapicuíba: Pró-fono; 2005. p. $175-83$.

16. Kuehn DP, Henne LJ. Speech Evaluation and Treatment for Patients With Cleft Palate. Am J Speech Lang Pathol. 2003;12(1):103-9.

17. Pegoraro- Krook MI, Dutka-Souza JCR, Magalhães LCT, Feniman MR. Intervenção fonoaudiológica na fissura palatina. In: Ferreira LP, Befi-Lopes DM, Limongi SCO, editors. Tratado de Fonoaudiologia. 1st ed. São Paulo: Rocca; 2004. p. $439-55$.

18. Bzoch KR. Introduction to the study of communicative disorders in cleft palate and related craniofacial anomalies. In: Bzoch KR, editor. Communicative disorders related to cleft lip and palate. 5th ed. Austin: pro-ed.; 2004. p. 3-66.

19. Kummer AW. Velopharyngeal dysfunction (VPD) and resonance disorders. In: Kummer AW. Cleft Palate and craniofacial anomalies: the effects on speech and resonance. San Diego: Singular Thomson Learning; 2001. p. $145-76$. 
20. Kummer AW, Briggs M, Lee L. The relationship between the characteristics of speech and velopharingeal gap size. Cleft Palate Craniofac J. 2003;40(6):590-6.

21. Laczi E, SussmanJE, Stathopoulos ET, Huber J. Perceptual evaluation of hypernasality compared to HONC measures: The role of experience. Cleft Palate Craniofac J. 2005 Mar;42(2):202-11.

22. Camargo LOS, et al. Oclusãovelofaríngea em indivíduos submetidos à nasoendoscopia na Clínica de Educação para Saúde (CEPS). Salusvita. 2001;20:35-47.

23. Netto ABC, Cervantes O. Comparative study among cleft patients with velopharyngeal insufficiency treated with speech therapy and pharyngoplasty. Rev. Bras. Cir. Plást. 2011;26:631-8.

24. Shyammohan A, Sreenivasulu D. Speech Therapy with Obturator, J Indian Prosthodont Soc. 2010;10:197-9.

25. Henningsson $G$, et al. Universal parameters for reporting speech outcomes in individuals with cleft palate. Cleft Palate Craniofac J. 2008;45:1-17.

26. Kummer AW. Speech therapy for errors secondary to cleft palate and velopharyngeal dysfunction. Semin Speech Lang. 2011;32:191-8.

27. Lam DJ, Starr JR, Perkins JA, Lewis CW, Eblen LE, Dunlap J, Sie KCY. A comparison of nasoendoscopy and multiview videofluoroscopy in assessing velopharyngeal insufficiency. Otolaryngol Head Neck Surg. 2006;134(3):394-402.

28. Fleiss JL. Statistical methods for rates and proportions. New York: John Wiley \& Sons; 1973.

29. Landis JR, Koch GG. The measurement of observer agreement for categorical data. Bometrics. 1977 Mar;33(1):159-74.

30. Altmann EBC, Lederman H. Videofluroscopia da Deglutição e do Esfíncter Velo-Faríngico: Padronização do Exame. Pró-fono Rev de Atual Cient. 1990;2:9-16.

31. Pulkkinen J, Ranta R, Heliövaara A, Haapanen M. Craniofacial characteristics and velopharyngeal function in cleft lip/palate children with and without adenoidectomy. Eur Arch Otorhinolaryngol. 2002;259:100-4.

32. Hardin-Jones MA, Brown CK, Van Demark DR, Morris HL. Long-term speech results of cleft palate patients with primary palatoplasty. Cleft palate Craniofac J. 1993;30:5563.
33. Kirschner RE, RandallP, Wang P, JawadA, Duran M, Huang $\mathrm{BAK}$, et al. Cleft palate repair at 3 to 7 months of age. Plastic and Reconstructive Surgery. 2000;105:2127-32.

34. Van Lierde KM, Monstrey S, Bonte K, Van Cauwenberge $\mathrm{P}$, Vinck B. The Long-term speech outcome in Flemish Young adults two different types of palatoplasty. Int $J$ of Pediat Oto. 2004;68:865-75.

35. Khosla RK, Mabry K, Charles L, Castiglione FACS. Clinical Outcomes of the Furlow Z-Plasty for Primary Cleft Palate Repair. Cleft Palate Craniofac J. 2008;45:501-10.

36. Genaro KF, Fukushiro AP, Suguimoto MLFCP. Avaliação e tratamento dos distúrbios da fala. In: Trindade IEK, Silva Filho OG. Fissuras Labiopalatinas: Uma abordagem Interdisciplinar. São Paulo: Santos; 2007. p. 109-22.

37. Trindade IEK, Genaro KF, Yamashita RP, Miguel HC, Fukushiro AP. Proposta de classificação da função velofaríngea na avaliação perceptivo-auditiva da fala. PróFono Revista de Atualização Científica. 2005;17:259-62.

38. John A, Sell D, Sweeney T, Harding-Bell A, Williams A. The Cleft palate audit for speech-augmented: a validated and reliable measure for auditing cleft speech. Cleft Palate Craniofac J. 2006;43:272-88.

39. Yoon PJ, Starr JR, Perkins JA, Bloom D, Sie KCY. Interrater and intrarater reliability in the evaluation of Velopharyngeal insufficiency within a single institution. Arch. Otolaryngol. Head Neck Surg. 2006;132:947-51.

40. Sie KCY, Starr JR, Bloom DC, Cummingham M, et al. Multicenter interrater and intrarater reliability in the endoscopic evaluation of Velopharyngeal insufficiency. Arch. Otolaryngol. Head Neck Surg. 2008;134:757-63.

41. Pegoraro-Krook MI, Dutka-Souza JCR, Marino VCC. Nasoendoscopy of Velopharynx before and during diagnostic therapy. J Appl Oral Sci. 2008;16:181-8.

42. Di Ninno CQMS, Rezende ALF, Jesus JDS, Pires JS, Godinho RN, Britto DBO. Caracterização do padrão de fechamentovelofaríngeo em pacientes com fissura palatina. Rev Soc Bras Fonoaudiol. 2012;17:161-6.

43. Penido FA, Noronha RM, Caetano KI, Jesus MS, Di Ninno CQ, Britto AT. Correlação entre os achados do teste de emissão de ar nasal e da nasofaringoscopia em pacientes com fissura labiopalatina operada. Rev Soc Bras Fonoaudiol. 2007;12:126-34.

44. Jesus MS, Penido FA, Valente P. Avaliações fonoaudiológicas clínica e instrumental em indivíduos com 
fissura labiopalatina. In: Jesus MS, Di Ninno CQ. Fissura labiopalatina: fundamentos para a prática fonoaudiológica. São Paulo: Roca; 2009.

45. Shi B. Consideration and treatment of clinical problems of lip cleft and palate cleft. Journal of Oral and Maxillofacial Surgery. 2005;15:125-7.

46. Qi C, Qian Z, Bing S, Heng Y, Tian M, Guang-ning Z. Study of relationship between clinical factors and velopharyngeal closure in cleft palate patients. J Res Med Sci. 2011;16:945-50.
47. Piccoli EMH. Hipernasalidade. In: Ferreira LP, editor. Um pouco de nós sobre voz. [place, publisher unknown]; 1992. p. 119-32.

48. Warren DW, Dalston RM, Mayor R. Hypernasality in the presence of "adequate" Velopharyngeal closure. CleftPalate Craniofac J. 1994;30:150-4.

49. Kuehn DP, Moon JB. Velopharyngeal closure force and levatorvelipalatini activation levels in varying phonetic contexts. Journal Speech, Language, and Hearing Research. 1998;41:51-62. 